\title{
The BglG group of antiterminators: a growing family of bacterial regulators
}

\author{
SUBRAMONY MAHADEVAN* \\ Developmental Biology and Genetics Laboratory, Indian Institute of Science, Bangalore \\ 560012, India
}

MS received 14 March 1997; revised 29 May 1997

\begin{abstract}
The product of the bglG gene of Escherichia coli was among the first bacterial antiterminators to be identified and characterized. Since the elucidation ten years ago of its role in the regulation of the $b g l$ operon of $E$. coli, a large number of homologies have been discovered in both Gram-positive and Gram-negative bacteria. Often the homologues of BglG in other organisms are also involved in regulating $\beta$-glucoside utilization. Surprisingly, in many cases, they mediate antitermination to regulate a variety of other catabolic functions. Because of the high degree of conservation of the cis-acting regulatory elements, antiterminators from one organism can function in another. Generally the antiterminator protein itself is negatively regulated by phosphorylation by a component of the phosphotransferase system. This family of proteins thus represents a highly evolved regulatory system that is conserved across evolutionarily distant genuses.
\end{abstract}

Keywords. Bacterial antitermination; RNA binding proteins; response regulators; phosphortransferase systems.

\section{Introduction}

Prokaryotic organisms have evolved a variety of mechanisms to regulate expression of their genes under diverse environmental conditions. Since most unicellular organisms do not have the luxury of cell differentiation, the single cell has to coordinate its response to arrays of signals deriving both from within and from outside. Bacteria probably evolved diverse regulatory mechanisms to suit the types of response that needed to be mounted to different types of stimuli.

Regulation by controlling elongation of transcription, a deviation from the gene regulation paradigm proposed by Jacob and Monod (1961), was first recognized in bacteriophages. The product of the $N$ gene of $\lambda$-phage was proposed to function as an antiterminator necessary for transcription to continue past sites of termination present in the phage genome (Roberts 1969). The presence or absence of the N protein makes termination "conditional". By incorporating additional elements that can modulate the efficiency of termination, the phage has thus evolved an ingenious mode of regulation using an element that is primarily a signal for termination of transcription. A possible advantage offered by this mode of regulation is that the kinetics of induction is likely to be fast since transcription initiation from the promoter, which is usually the rate limiting step in gene expression, has already been accomplished. The virus can therefore respond quickly to the inducing signal. In this case the response relates to

\footnotetext{
*Fax, (080)3341683; Email, mahi@serc.iisc.ernet.in.
} 
a major developmental decision between lysis and lysogeny where precise timing of the genetic switch is of paramount importance. The biochemical and molecular aspects of the antitermination reaction mediated by the $\mathrm{N}$ protein (which requires a specific cis-acting element and several other host-coded proteins) have been intensely studied. $\mathrm{N}$-mediated antitermination offers a paradigm for regulation by antitermination (for a recent review, see Greenblatt et al 1993).

Does such a mechanism exist in the case of chromosomal genes? There are many situations in which regulation by antitermination of chromosomal genes is implicated, for instance for the ribosomal RNA genes (reviewed by Morgan 1986). But the closest example of regulation by antitermination involves the amino acid biosynthetic Operons of E. coli, where the regulation is by attenuation of transcription (Yanofsky 1981). Here, termination is modulated by the coupling of transcription to translation. This occurs in such a way that under conditions of amino acid starvation, ribosomes stall within an open reading frame in the leader transcript, resulting in the formation of an alternative secondary structure that preempts termination within the leader. The presence of the respective amino acid results in movement of ribosomes across the mRNA, causes the formation of the terminator structure and blocks transcription elongation.

A modified form of regulation by attenuation was documented in the case of the trp operon of B. subtilis. In B. subtilis, the role played by ribosomes in regulating transcription termination in the $E$. coli system was attributed to an operon-specific regulator that was postulated to act by binding to the nascent message in a tryptophandependent fashion (Shimotsu et al 1986). The leader mRNA can assume alternative secondary structures and the binding of the protein to the leader sequence in the presence of tryptophan will favour the formation of a terminator structure. In the absence of tryptophan, an alternative secondary structure would preempt the formation of the terminator structure, leading to read-through of transcription. In a similar manner, a positive regulator was postulated to control expression of $\operatorname{sacB}$ (a gene encoding an excreted levansucrase in B. subtilis) by antitermination of transcription in an operon-specific manner (Shimotsu and Henner 1986). In both cases, the regulatory proteins were subsequently identified (Babitzke et al 1996, references cited below).

During the past ten years, several reports of studies in E. coli and B. subtilis have provided additional examples for regulation of bacterial gene expression by antitermination of transcription. The interesting feature of these studies is that they have helped to identify a closely related family of antiterminators that bring about regulation by directly interacting with mRNA. The activity of the antiterminator is modulated by a specific component of the phosphotransferase system (PTS) in most cases. The cis- and transacting elements of the system are conserved across evolutionarily distant organisms.

Regulation by antitermination in catabolic Operons was recently examined by Rutberg (1997) in an article which appeared after the present review was well under way. Here I present the evolution of concepts and some of the key experimental approaches that led to our understanding of the mechanisms involved in regulation by antitermination mediated by the BglG class of regulators.

\section{Antitermination in the bgl operon of $E$. coli}

The $b g l$ operon comprises a set of genes involved in the catabolism of aryl- $\beta$-glucosides in $E$. coli. It has received considerable attention because of the unique regulatory 
mechanism involved in its expression. Wildtype $E$. coli strains carry the $b g l$ genes in an intact form, but surprisingly exhibit a $\mathrm{Bgl}^{-}$phenotype. Spontaneous mutations can arise and result in the activation of the operon, rendering the strains $\mathrm{Bgl}^{+}$. The predominant class of such activating mutations consists of insertions of DNA upstream of the silent promoter leading to the enhancement of transcription from the promoter (Reynolds et al 1981, 1986). Once the promoter is transcriptionally activated, optimal expression requires the presence of $\beta$-glucoside substrates.

Genetic analysis of the operon had suggested the presence of a positive regulator (Prasad and Schaefler 1974). Studies using transcriptional fusions indicated that the first gene of the operon, $b g l G$ (the nomenclature of the gene has been changed from $b g l C$ to $b g l G$ ), is necessary for the expression of the operon (Mahadevan et al 1987). Expression of a $b g l-l a c Z$ transcriptional fusion was constitutive in a strain that expressed only the $b g l G$ gene. However, presence of the second gene of the operon, $b g l F$, along with $b g l G$, resulted in expression dependent on the presence of $\beta$-glucosides; this suggested a negative role for BglF in regulation (Mahadevan et al 1987). The finding was made more interesting by the observation that $b g l F$ encodes the putative $b g l$ specific component of the phosphotransferase system and $b g l F$ mutants are phenotypically $\mathrm{Bgl}^{-}$. Thus, BglF apparently has both permease as well as regulatory activities.

Analysis of $b g l$ regulation in plasmid constructs in which the $b g l$ promoter was replaced by a vector promoter showed that expression of the genes is still subject to induction by $\beta$-glucosides (Mahadevan and Wright 1987). This gave the first indication that the positive regulation mediated by $\mathrm{BglG}$ is occurring at sites different from the site of initiation of transcription. A systematic search for the regulatory site showed that a fusion of the reporter gene at position +31 led to BglG-independent expression. Nucleotide sequence analysis of the $b g l$ leader region showed the presence of a $\rho$ independent terminator which was functional in vitro at an efficiency greater than $90 \%$ (Mahadevan and Wright 1987). Mutations within the terminator resulted in partial BglG independence in vivo and led to reduced termination in vitro. In addition, Overexpression of the $b g l$ leader transcript in a strain carrying a single copy of the $b g l G$ gene resulted in lowering of transcription from a $b g l-l a c Z$ fusion. These results led to a model for the regulation of the operon in which BglG was postulated to act as a positive regulator by functioning as an antiterminator, most likely by directly binding to the leader RNA. BglF was postulated to function as a negative regulator by inactivating BglG in the absence of the inducer (Mahadevan and Wright 1987).

Determination of the nucleotide sequence of the operon by Schnetz et al (1987) showed the presence of another terminator located within the inter-cistronic region between $b g l G$ and $b g l F$. BglG was subsequently shown to mediate regulation at the downstream terminator also (Schnetz and Rak 1988). Comparison of the nucleotide sequence in the vicinity of the two terminators showed the presence of a 38 nucleotide sequence common to the two terminators and partially overlapping with the inverted repeats of the terminator (Schnetz et al 1987). Surprisingly, the same motif was also seen in the case of two $B$. subtilis genes which were thought to be regulated by antitermination, the $s a c B$ gene that encodes an excreted levansucrase and a gene that encodes an excreted $\beta$-endoglucanase.

In a series of elegant experiments, Houman et al (1990) demonstrated the direct interaction of labelled $b g l$ leader RNA, expressed from a T7 promoter, with purified $\mathrm{BglG}$ protein bound to membrane. The specific sequence of the $b g l$ leader involved in binding to $\mathrm{BglG}$ was determined by monitoring protein-RNA interaction after 
hybridizing the leader RNA to a series of oligonucleotides spanning the terminator region. Based on an analysis of mutants that abolished $\mathrm{BglG}$ binding, the target for binding was conjectured to lie within a 32-nucleotide region extending from position +41 to +72 . This sequence has the potential to assume a hairpin structure that is probably stabilized by the binding of BglG. Mutations that have drastic effects on $\mathrm{BglG}$ binding are located within the stem region of the hairpin. Interestingly, the second terminator in the operon, located between the $b g l G$ and $b g l F$ genes, differs at four positions, two within the loop region and two within the stem region of the hairpin. The mutations in the stem region convert an A-T base pair to a $\mathrm{G}-\mathrm{C}$ base pair without affecting the secondary structure. It is not clear at present whether the changes in the sequence quantitatively affect the regulation mediated by $\mathrm{BglG}$ at the two terminators. This possibility could be tested using reporter gene constructs.

The nature of negative regulation mediated by $\mathrm{BglF}$ was investigated by AmsterChoder et al (1989). Using extracts containing soluble components of the PTS, it was demonstrated that the BglF protein is phosphorylated in the presence of phospho-enolpyruvate (PEP). This phosphate group can be transferred to the substrate $\beta$-glucoside or the $\mathrm{BglG}$ protein in a mutually exclusive manner. Interestingly, when $\mathrm{BglG}$ mutants that show constitutive phenotype in vivo were used in the assay, very low levels of phosphorlyation was detected. These observations led to the proposal that BglF negatively regulates the $b g l$ operon by phosphorylating the antiterminator in the absence of the substrate. Similar observations were subsequently reported by Schnetz and Rak (1990). It is rare to come across other examples of a regulatory protein phosphorylating such dissimilar substrates, a protein and a carbohydrate in this case.

Using fusions of the BglG protein to the DNA binding domain of $\lambda$-repressor, the BglG protein was shown to function as a dimer (Amster-Choder and Wright 1992). However, in the absence of the substrate, phosphorylation of the BglG subunits resulted in dissociation and subsequent loss of DNA binding. This demonstration provided the molecular basis for the negative action of the BglF protein. Thus the BglG-BglF combination is an efficient two-component response regulator with a membrane-bound sensor molecule and a cytoplasmic regulator which functions as an antiterminator. The interaction between the two components involves phosphorylation and is modulated by the presence of $\beta$-glucosides (figure 1).

\section{BglG homologues in $B$. subtilis}

Are there counterparts to the BglG-BglF regulators in other prokaryotic systems? The nucleotide sequence analysis of the operon (Schnetz et al 1987) had already indicated the possibility of regulation by a similar mechanism in two B. subtilis systems. The cis-acting elements involved in antitermination in the $E$. coli bgl operon share considerable similarity with the putative regulatory regions of two B. subtilis genes, one encoding an excreted levansucrase gene (Shimotsu and Henner 1986; Steinmetz et al 1985) and another encoding an excreted $\beta$-endoglucanase (Murphy et al 1984). A partial open reading frame preceding the $\beta$-endoglucanase gene shared $38 \%$ identity with the $85 \mathrm{C}$-terminal residues of the $\mathrm{BglG}$ antiterminator suggesting a similar role for the $B$. subtilis protein in regulation. Both sucrose utilization and glucan catabolism in $B$. subtilis were shown to involve regulation by antitermination. 
Uninduced

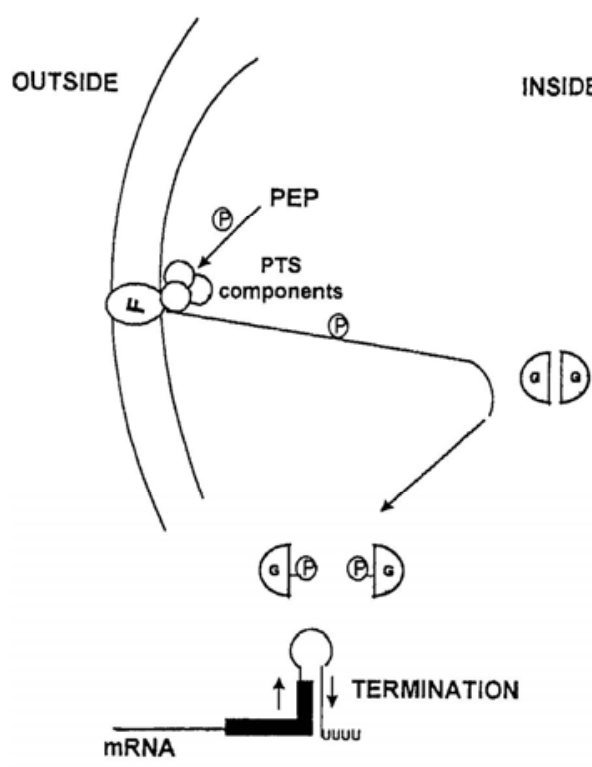

\section{Induced}

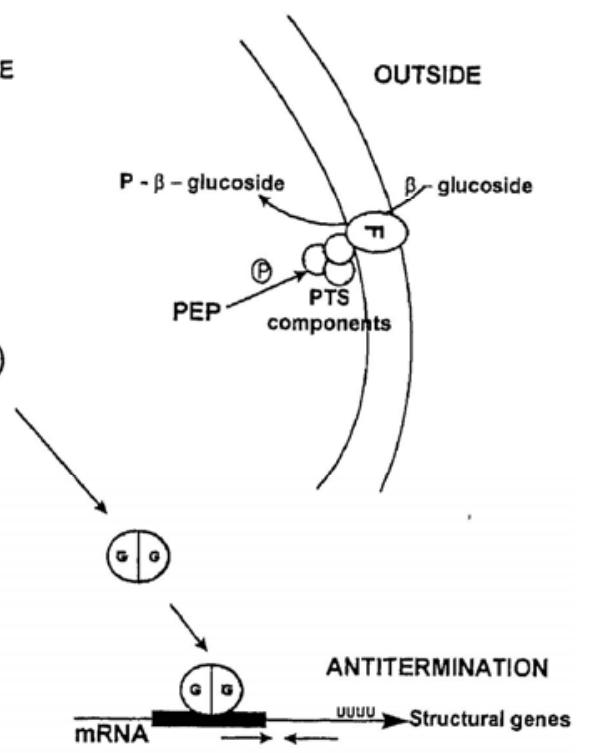

Figure 1. Regulation by antitermination in the $b g l$ operon of E. coli. The shaded box represents the canonical sequence recognized by the unphosphorylated BglG homodimer. Absence of the inducer results in the phosphorylation of BglG leading to termination. See text for details. (Based on Mahadevan and Wright 1987; Amster-Choder et al 1989; Schnetz and Rak 1990; Houman et al 1990; Amster-Choder and Wright 1992).

In addition to the excreted levansucrase gene $s a c B$, the $s a c P A$ genes of $B$. subtilis encode an intracellular phosphosucrase and a sucrose-specific component of the PTS (Fouet et al 1987). Recent reports indicate that the two sucrose utilizing systems have independent antiterminators, SacY and SacT. SacY is involved in regulating $s a c B$ and SacT regulates sacPA expression (Steinmetz et al 1989; Crutz et al 1990; Debarbouille et al 1990). Under specific conditions, each of the two antiterminators can mediate regulation of both systems since the cis-acting elements share a high degree of homology (Steinmentz et al 1989). Interestingly, subtle differencees in the sequence of the cis-elements present near the two terminators confer specificity to the respective antiterminator (Aymerich and Steinmetz 1992). Since expression of sacB requires higher concentrations of sucrose compared to that needed to induce the intracellular sucrase SacA, differential regulation of the two systems is likely to be modulated through the PTS in response to sucrose concentrations. This may ultimately depend on the ecological niche of the organism (Steinmetz et al 1989).

Analysis of glucan utilization in B. subtilis by Schnetz et al (1996) indicated the presence of two genes, licS which codes for an $\beta$-endoglucanase and the regulatory gene licT. Though induction of licS by lichenan is only two-fold, licT is essential for growth on lichenan as the sole carbon source. Because of the high degree of similarity of the cis-acting elements, LicT expressed in E. coli can cause antitermination in the $b g l$ operon. Similarly, BglG can result in antitermination at the licS terminator. However, it is not clear whether BglF can exert negative regulation of LicT. 
Recent studies show that $B$. subtilis also encodes a $\beta$-glucoside utilization system (Le Coq et al 1995). The B. subtilis bgl system consists of two genes, $b g l H$ and $b g l P$. The $b g l H$ gene codes for a phospho $\beta$-glucosidase and the $b g l P$ gene encodes a protein with homology to the $E$. coli $\mathrm{BglF}$ protein, the bgl-specific component of the PTS. Interestingly, the $b g l$ system in B. subtilis does not encode a BglG homologue though the $b g l P$ gene is preceded by a terminator with sequences similar to those seen in $E$. coli and B. subtilis terminators responding to the BglG class of antiterminators. LicT is involved in regulation by antitermination at this site (Kruger and Hecker 1995). Thus a regulator encoded by an operon involved in the metabolism of lichenan is also involved in regulating the expression of the genes pertaining to the catabolism of $\beta$-glucosides, an apparently unrelated metabolite. Though LicT regulates both licS and $b g l P H$, only licS is inducible by lichenan and not $b g l P H$. However, ßglucosides can induce both systems. Thus, the mechanism of induction is different for the two systems. This may be on account of differences at the level of either the proteins interacting with LicT or the cis-acting elements present in the two systems, or both.

Genetic evidence also indicates that LicT can substitute for SacY and SacT in strains deleted for the genes coding for the two antiterminators. These strains regain the ability for sucrose utilization when $b g l P$ is mutated; however, this suppression is lost when licT carries mutations (Le Coq et al 1995). Since bglP negatively regulates lict it will be interesting to see if licS is constitutive in a bglP mutant. Thus B. subtilis has at least three $\mathrm{BglG}$ homologues that can substitute for each other under specific conditions (figure 2).

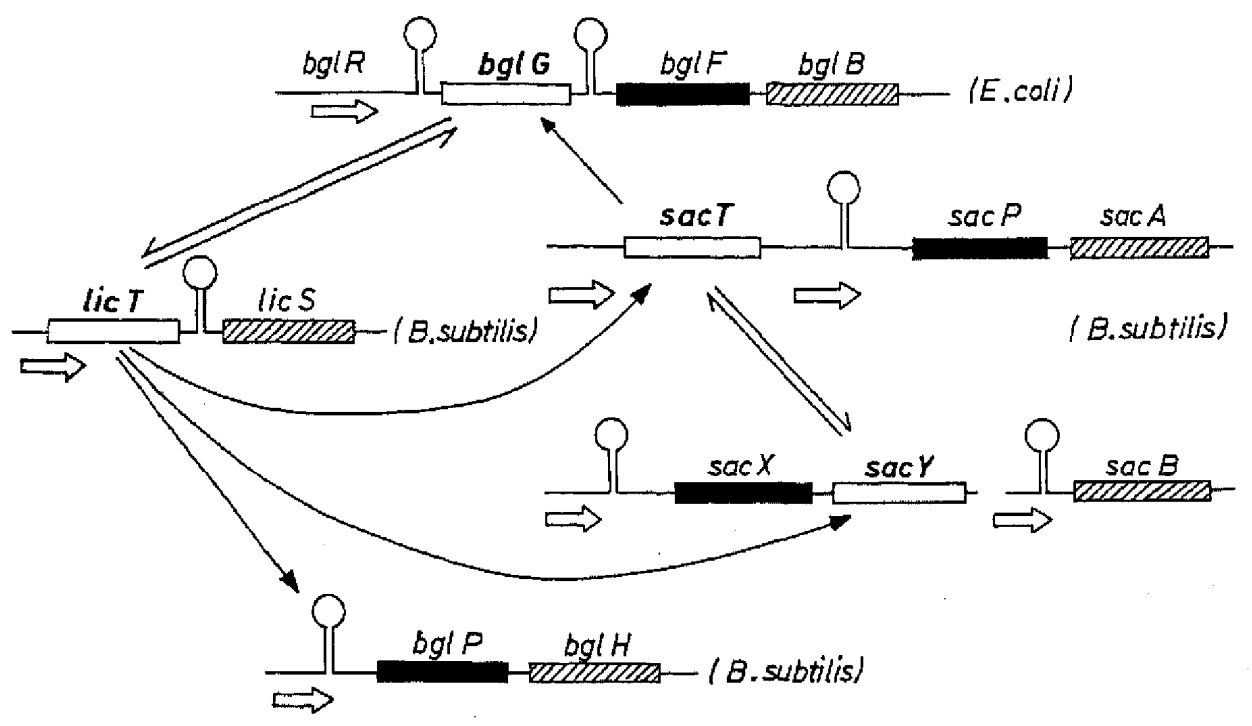

Figure 2. Cross-talk among the different catabolic systems in E. coli and B. subtilis. The genes encoding the antiterminators are indicated in bold letters. The shaded boxes represent PTS components that usually act as negative regulators. Horizontal arrows indicate direction of transcription. The thin arrows represent cross-regulation between the antiterminators. 


\section{BglG homologues in other systems}

Metabolism of $\beta$-glucosides in Lactococcus lactis is regulated by BglR, a homologue of BglG. BglR can prevent termination at the E. coli terminator tl (Bardowski et al 1994). Interestingly, the only other member of the Enterobacteriaceae that has been shown to have a BglG homologue is the Phytopathogenic bacterium Erwinia chrysanthemi. The ArbG protein of E. chrysanthemi shares considerable homology with the BglG family of proteins suggesting a similar role for ArbG in regulating the arb operon (El Hassouni et al 1992). In the near future, this family of antiterminators is likely to grow with the discovery of similar systems in other organisms.

\section{Summary and conclusions}

The combination of a terminator carrying a canonical cis element, regulated by a two component system consisting of an antiterminator and a PTS permease, appears to be a highly evolved system that is conserved in a wide variety of microorganisms. There are at least three bglG homologues in B. subtilis and they can functionally substitute for one another even though they regulate diverse metabolic functions. Even among those that regulate similar catabolic steps such as SacY and SacT that regulate sucrose metabolism, there are subtle differences in the cis and the trans elements enabling the two antiterminators to respond to different inducing signals and regulate the two different sucrases. Strangely, the B. subtilis $b g l$ operon has to "borrow" the antiterminator from the lic system though it has the canonical cis regulatory elements. Interestingly, even with a single antiterminator, differential regulation of the $\beta$ endoglucanase and the phospho- $\beta$-glucosidase genes is achieved. This may be related to the differences in the interaction with the PTS components or because of the intrinsic differences in the cis-acting elements. Regulation of the functionally similar antiterminators in the cell in response to the availability of different substrates is therefore a complex process.

The question as to why B. subtilis has this large pool of antiterminators with identical target sequence is puzzling. In addition, since most of the genetic systems characterized involve normal catabolic functions, the advantage of regulation by antitermination, as opposed to the conventional mode of negative regulation involving a repressor, is also unclear. One possibility is that global regulation of a large number of catabolic functions by a primary catabolite such as glucose is possible because of the involvement of the phosphotransferase system in regulation.

It is also interesting that barring E. coli and E. chrysanthemi, no report of a homologue is available in Gram-negative organisms. Whether this is due to lack of information about other systems or a genuine aspect of bacterial evolution, only future studies can tell.

An important observation regarding regulation by antitermination in E. coli and other organisms examined is that the $E$. coli $b g l$ operon is cryptic in spite of the presence of a highly sophisticated regulatory system. This aspect of the E. coli bgl operon has remained an evolutionary puzzle. A possible answer to this puzzle is that the antitermination function of $\mathrm{BglG}$ is recalled periodically for the regulation of other genetic system(s) in the organism which may carry the cis-acting regulatory elements. As a result, though there is no direct selection for the $\beta$-glucoside utilization function, selective 
pressure may be operating to maintain the regulatory functions of BglG and BglF. The low basal level of expression from the silent promoter is probably sufficient to regulate the expression from the unknown gene(s) located elsewhere on the E. coli chromosome. In the light of the cross-regulation seen in B. subtilis, this is an attractive possibility. However, in the absence of any direct evidence, it remains speculative. Identification of a candidate gene elsewhere on the chromosome will be a step in the right direction to substantiate this possibility.

In summary, the BglG class of antiterminators are widely distributed among prokaryotes. They constitute a major component of an evolutionarily conserved regulatory system involved in signal transduction and transcriptional regulation. Molecular characterization of their interactions with the cis- and trans-acting elements of the regulatory system is expected to enrich our understanding of two important biological processes, signal transduction and control of gene expression.

\section{Acknowledgements}

I wish to thank M Mukerji for the comments on the manuscript and members of the laboratory for fruitful discussions.

\section{References}

Amster-Choder O, Houman F and Wright A 1989 Protein phosphorylation regulates transcription of the $\beta$-glucoside utilisation operon in E. coli; Cell 58 847-858

Amster-Choder O and Wright A 1992 Modulation of the dimerisation of a transcriptional antiterminator protein by phosphorylation; Science 257 1395-1398

Aymerich S and Steinmetz M 1992 Specificity determinants and structural features in the RNA targets of the bacterial antiterminator proteins of the BglG/SacY family; Proc. Natl. Acad. Sci. USA 89 10410-10414

Babitzke P, Yealy J and Campanelli D 1996 Interaction of the trp RNA-binding attenuator protein (TRAP) of Bacillus subtilis with RNA: the efects of the number of GAG repeats, the nucleotides separating the adjacent repeats, and RNA secondary structure; J. Bacteriol. 178 5159-5163

BardowskiJ, Ehrlich S D and Chopin A1994 The BglR protein, which belongs to the BglG family of proteins, is involved in $\beta$-glucoside utilisation in Lactococcus lactis; J. Bacteriol. 176 5681-5685

Crutz A M Steinmetz M Aymerich S, Richter R and Le Coq D 1990 Induction of levansucrase in Bacillus subtilis: an antitermination mechanism negatively controlled by the phosphotransferase system; $J$. Bacteriol. 172 1043-1050

Debarbouille M, Arnaud M. Fouet A, Klier A and Rapoport G 1990 The sacT gene regulatnig the $\operatorname{sacPA}$ operon in Bacillus subtilis shares strong homology with transcriptional antiterminators; J. Bacteriol. 172 3966-3973

El Hassouni M, Henrissat B, Chippaux M and Barras F 1992 Nucleotide sequence of the arb genes which control $\beta$-glucoside utilisation in Erwinia chrysanthemi: comparison with the Escherichia coli bgl operon and evidence for a new $\beta$-glycohydrolase family including enzymes from eubacteria, archebacteria, and humans; J. Bacteriol. 174 765-777

Fouet A, Arnaud M, Klier A and Rapoport G 1987 Bacillus subtilis sucrose specific enzyme II of the phosphotransferase system: expression in Escherichia coli and homology to other enzymes II from enteric bacteria; Proc. Natl. Acad. Sci. USA 84 8773-8777

Greenblatt J, Nodwell J R and Mason S W 1993 Transcriptional antitermination; Nature (London) 364 401-407

Houman F, Diaz-Torrers M R and Wright A 1990 Transcriptional antitermination in the $b g l$ operon of $E$. coli is modulated by a specific RNA binding protein; Cell 62 1153-1163

Jacob F and Monod J 1961 Genetic regulatory mechanisms in the synthesis of proteins; J. Mol. Biol. 3 318-356 
Kruger S and Hecker M 1995 Regulation of the putative bglPH operon for aryl $\beta$-glucoside utilization in Bacillus subtilis; J. Bacteriol. 177 5590-5597

Le Coq D, Lindner C, Kruger S, Steinmetz M and Stulke J 1995 New $\beta$-glucoside (bgl) genes of Bacillus subtilis: the $b g l P$ gene product has both transport and regulatory functions similar to those of $\mathrm{BglF}$, its $E$. coli homologue; J. Bacteriol 177 1527-1535

Mahadevan S, Reynolds A E and Wright A 1987 Positive and negative regulation of the $b g l$ operon of E. coli; J. Bacteriol. 169 2570-2578

Mahadevan S and Wright A 1987 A bacterial gene involved in transcription antitermination: regulation at a rho-independent terminator in the $\mathrm{bgl}$ operon of E. coli; Cell $\mathbf{5 0} 485-494$

Morgan E A 1986 Antitermination mechanisms in rRNA Operons of Escherichia coli; J. Bacteriol. 168 1-5

Murphy N, McConnel D J and Cantwell B A 1984 The DNA sequence of the gene and the genetic control sites for the excreted B. subtilis enzyme $\beta$-glucanase; Nucleic Acids Res. 12 5355-5367

Prasad I and Schaefier S 1974 Regulation of the $\beta$-glucoside system in Escherichia coli K - 12; J. Bacteriol. 120 638-650

Reynolds A E, Felton J and Wright A 1981 Insertion of DNA activates the cryptic $b g l$ operon of E. coli K12; Nature (London) 293 625-629

Reynolds A E, Mahadevan S, LeGrice S F J and Wright A 1986 Enhancement of bacterial gene expression by insertion elements or by mutation in a CAP-cAMP binding site; J. Mol Biol. 191 85-95

Roberts J W 1969 Termination factor for RNA synthesis; Nature (London) 224 1168-1174

Rutberg B 1997 Antitermination of transcription in catabolic operons; Mol. Microbiol. 23 413-421

Schnetz K, Toloczyki C and Rak B 1987 B-glucoside (bgl) operon of Escherichia coli K12: nucleotide sequence, genetic organisation, and possible evolutionary relationship to regulatory components of two Bacillus subtilis genes; J. Bacteriol 169 2579-2590

Schnetz K and Rak B 1988 Regulation of the $b g l$ operon of Escherichia coli by transcriptional antitermination; EMBO J. 7 3271-3277

Schnetz K and Rak B $1990 \beta$-glucoside permease represses the $b g l$ operon of $E$. coli by phosphorylation of the antiterminator protein and also interacts with enzymeIII ${ }^{\text {glc }}$, the key element in catabolite control; Proc. Natl. Acad. Sci. USA 87 5074-5078

Schnetz K, Stulke J, Gertz S, Kruger S, Krieg M, Hecker M and Rak B 1996 LicT, a Bacillus subtilis transcriptional antiterminator protein of the BglG family; J. Bacteriol. 178 1971-1978

Shimotsu H, Kuroda M I, Yanofsky C and Henner D 1986 Novel form of transcriptional attenuation regulates expression of the Bacillus subtilis tryptophan operon; J. Bacteriol 166 461-471

Shimotsu H and Henner D 1986 Modulation of Bacillus subtilis levansucrase gene expression by sucrose and regulation of the steady state mRNA level by sac $U$ and sacQ genes; J. Bacteriol. 168 380-388

Steinmetz M, Le Coq D and Aymerich S 1989 Induction of saccharolytic enzymes by sucrose in Bacillus subtilis: evidence for two partially interchangeable pathways; J. Bacteriol. 171 1519-1523

Steinmetz M, Le Coq D and Aymerich S, Gonzy-Tréboul G and Gay P 1985 The DNA sequence of the gene for the secreted Bacillus subtilis enzyme levansucrase and its genetic control sites; Mol. Gen. Genet. 200 220-228

Yanofsky C1981 Attenuation in the control of expression of bacterial operons; Nature (London) 289 751-758 